\title{
Brain Plasticity and Rehabilitation in Stroke Patients
}

\author{
Yukihiro Hara
}

The Department of Rehabilitation Medicine, Nippon Medical School

\begin{abstract}
In recent years, our understanding of motor learning, neuroplasticity and functional recovery after the occurrence of brain lesion has grown significantly. Novel findings in basic neuroscience have provided an impetus for research in motor rehabilitation. The brain reveals a spectrum of intrinsic capacities to react as a highly dynamic system which can change the properties of its neural circuits. This brain plasticity can lead to an extreme degree of spontaneous recovery and rehabilitative training may modify and boost the neuronal plasticity processes. Animal studies have extended these findings, providing insight into a broad range of underlying molecular and physiological events. Neuroimaging studies in human patients have provided observations at the systems level that often parallel findings in animals. In general, the best recoveries are associated with the greatest return toward the normal state of brain functional organization. Reorganization of surviving central nervous system elements supports behavioral recovery, for example, through changes in interhemispheric lateralization, activity of association cortices linked to injured zones, and organization of cortical representational maps. Evidence from animal models suggests that both motor learning and cortical stimulation alter intracortical inhibitory circuits and can facilitate long-term potentiation and cortical remodeling. Current researches on the physiology and use of cortical stimulation animal models and in humans with stroke related hemiplegia are reviewed in this article. In particular, electromyography (EMG)-controlled electrical muscle stimulation improves the motor function of the hemiparetic arm and hand. A multi-channel near-infrared spectroscopy (NIRS) studies in which the hemoglobin levels in the brain were non-invasively and dynamically measured during functional activity found that the cerebral blood flow in the injured sensory-motor cortex area is greatest during an EMG-controlled FES session. Only a few idea is, however, known for the optimal timing of the different processes and therapeutic interventions and for their interactions in detail. Finding optimal rehabilitation paradigms requires an optimal organization of the internal processes of neural plasticity and the therapeutic interventions in accordance with defined plastic time windows. In this review the mechanisms of spontaneous plasticity after stroke and experimental interventions to enhance plasticity are summarized, with an emphasis on functional electrical stimulation therapy. (J Nippon Med Sch 2015; 82: 4-13)
\end{abstract}

Key words: stroke, functional electrical stimulation, brain, plasticity, rehabilitation

\section{Introduction}

For long time it has been believed that the hardware of the brain is exactly "hard", that once an incident such as stroke happens, brain structures and functions are lost forever. Clinically, the most successful therapy to further enhance functional recovery is rehabilitative training. Rehabilitation as a term "to reach and maintain optimal functioning in physical, intellectual, psychological and/or social domains" (WHO. International classification of functioning disability Health ICF. Geneva: WHO; 2001) is evidence based medicine and does not exclude a specific subgroup of patients. More understanding of clinical recovery has been accompanied by a better understanding of its neurobiological basis. The improved function is associated with remodeling in remaining intact cortical structures within the brain as has been demonstrated to occur in adult mammalian animal models following brain injury ${ }^{1,2}$. This line of research has enlightened us on

Correspondence to Yukihiro Hara, MD, PhD, Department of Rehabilitation Medicine, Nippon Medical School Chiba Hokusoh Hospital, 1715 Kamakari, Inzai, Chiba 270-1694, Japan

E-mail: hara-y@nms.ac.jp

Journal Website (http://www.nms.ac.jp/jnms/) 
the effects of rehabilitation training on central nervous system physiology and has led to the well-accepted principle that neural recovery is dependent on active skilled use $^{3-5}$. It has also demonstrated cortical motor map reorganization as a result of skilled arm and hand training in primates, suggesting that similar neuroplastic mechanisms are involved in motor learning. Plasticity mechanisms include activity-dependent rewiring and synapse strengthening. The challenge for improving stroke recovery is to understand how to optimally engage and modify surviving neuronal networks, to provide new response strategies that compensate for tissue lost to injury $^{6}$. Enhancing stroke recovery by facilitating brain plasticity with the direct application of a physical modality to the cerebral cortex is a relatively new area of investigation in rehabilitation and neuroscience.

\section{Brain Plasticity and Rehabilitation in Animals}

Takatsuru et al. showed that novel neuronal circuits mediating the response to sensory vibratory stimulation of the impaired limbs developed in the intact somatosensory cortex over the first weeks after focal infarction and became established by 4 weeks by using current source density analysis technique ${ }^{7}$. F-MRI studies concentrating on the affected upper limb in rats have described a shift in laterality of activation after stroke such that early after stroke, brain activation during affected paw stimulation is mainly in the contralesional cortex, later after stroke activity shifts toward the normal pattern, that is the ipsilesional cortex ${ }^{8-10}$ found that the larger the ischemic insult the stronger the activity in the contralesional M1 (primary motor cortex). In accordance with human studies van Meer et al. ${ }^{11}$ could show that functional recovery after middle cerebral artery occlusion in rats was correlated with the extent of preservation or restoration of the ipsilesional corticospinal tract in combination with reinstatement of inter-hemispheric neuronal signal synchronization and normalization of focal network organization. Using different tracing techniques ${ }^{12}$, could show which neurons take over when functional map shifts occur: if the forelimb M1 in rats was destroyed, neurons in the hindlimb area took over to enable functional recovery of the forelimbs. This functional shift was based on sprouting of new axon branches from hindlimb corticospinal fibers into the cervical spinal cord, followed by retraction of the original lumbar projecting axon and thus a conversion of a hindlimb into a forelimb projecting neuron. The most significant gains in the recovery of forelimb reaching ability were achieved when rehabilitation was initi- ated early, (i.e., 5 days) after stroke as compared to 14 and 30 days after stroke. Recovery was associated with increased dendritic branching of layer V M1 neurons in the unlesioned hemisphere- a response that was not detected when rehabilitation was delayed by 30 days ${ }^{13}$. Wahl et al. ${ }^{14}$ showed nearly full recovery of skilled forelimb functions in rats with large strokes when a growthpromoting immunotherapy against a neurite growthinhibitory protein was applied to boost the sprouting of new fibers, before stabilizing the newly formed circuits by intensive training. In contrast, early high-intensity training during the growth phase destroyed the effect and led to aberrant fiber patterns. Pharmacogenetic experiments identified a subset of corticospinal fibers originating in the intact half of the forebrain, side-switching in the spinal cord to newly innervate the impaired limb and restore skilled motor function ${ }^{14}$. They suggested the presence of critical time windows during which the brain is most responsive to the application of growthpromoting agents and to training-dependent plasticity ${ }^{14}$. Serial functional MRI (fMRI) studies examining the affected upper limb in rats have described a shift in laterality of activation after stroke such that early after stroke, brain activation during affected paw stimulation is mainly ipsilateral, that is, in the contralesional sensorimotor cortex; later after stroke, activity shifts toward the normal pattern, being contralateral, that is, in the ipsilesional sensorimotor cortex ${ }^{8,9}$. These findings are closely aligned with fMRI studies in humans ${ }^{15,16}$. Van Meer et al. ${ }^{11}$ advocated the four points about brain plasticity after stroke.: (1) improvement of sensorimotor function correlates with restoration of interhemispheric functional connectivity and normalization of network configuration of the bilateral sensorimotor cortex, (2) gradual recovery of sensorimotor function after a large stroke correlates with increased structural integrity of the ipsilesional corticospinal tract, (3) restoration of inter-hemispheric functional connectivity between bilateral sensorimotor cortices is associated with unilateral improvement of structural integrity of the ipsilesional corticospinal tract, and (4) good functional outcome is linked with preserved or repaired structural integrity within the ipsilesional corticospinal tract.

Other changes can arise in injured areas distant from stroke, including diaschisis. Diaschisis refers to reduced activity, typically measured by blood flow and/or metabolism, in uninjured brain areas that have rich connections with injured brain areas ${ }^{17}$. In some studies, behavioral recovery is related to resolution of diaschisis, that is, 
restitution of brain activity in these uninjured areas that are distant from, but connected to, the site of infarct ${ }^{16,18,19}$. Post infarct rehabilitative training rapidly improves motor performance and movement quality after an ischemic infarct in motor cortex. However, training-induced motor improvements are not reflected in spared motor maps until substantially later, suggesting that early motor training after stroke can help shape the evolving poststroke neural network ${ }^{20}$.

\section{Brain Plasticity and Rehabilitation in Humans Brain Plasticity Revealed by Functional Neuroimag- ing}

Recent advances in functional imaging of human brain activity in stroke patients, (positron emission tomographic (PET) and fMRI), reveal that cortical hemisphere contralateral to the infarction lesion plays an important role in this recovery process ${ }^{21,22}$. There is also clinical evidence showing that the post ischemic reorganization occurring in somatosensory system of the contralesional (intact) hemisphere plays an important role for compensation for impaired functions ${ }^{23}$. The underlying mechanism of this compensation occurring in the intact hemisphere is important for optimizing the functional recovery of human stroke patients ${ }^{21}$. The brain, including the motor system, learns by repetition and training. Many basic mechanisms, however, are still poorly understood, and rehabilitative training is largely evidencebased medicine ${ }^{24}$. Nevertheless there are no generally accepted guidelines and no definite recommendations concerning the timing, kind and intensity of stroke rehabilitation $^{25}$. Stroke recovery is a complex process that probably occurs through a combination of restoration, substitution and compensation of functions. That is why it has been also difficult to translate results from rehabilitative studies in animals to recommendations for rehabilitative schedules in human stroke patients. A majority of clinical studies has been conducted in chronic stroke patients $(>6$ months after the stroke) as recruitment of these patients was easier and baseline performance had stabilized ${ }^{26}$. These circumstances lead to functional outcome measurements probably gained largely from compensatory techniques to improve skills for daily living. The time courses of motor recovery differ among animal and human studies: While recovery in rodent models reaches the maximum around 4 weeks after stroke, human stroke survivors complete most of their recovery within 3 months ${ }^{26,27}$. NIRS could be used to predict the potential for clinical improvement in chronic stroke patients. It is also the first to demonstrate the complementary nature of neurophysiological and imaging techniques with NIRS in the prediction of functional potential and clinical outcomes. These findings have implications for clinical decision-making. Evaluation of brain function, using a combination of neurophysiological measures and imaging, can inform the setting of therapeutic effectiveness and the selection of patients for particular rehabilitation programs. This may lead to the conceptualization of rehabilitation strategies that are designed to maximally enhance rehabilitation through tailoring to individual patient deficits. Rehabilitation strategies may now be designed and optimized by employing methods to synchronize functional training of brain regions undergoing neural plasticity. However, a larger sample size, longer duration of training, or a restricted inclusion of stroke location and volume may be needed to demonstrate a difference between individually tailored rehabilitation programs and generic rehabilitation programs in efficacy in producing behavioral changes. Therapeutic approaches which directly stimulate the peripheral nerve system or central nerve system electrically or by magnetic pulses may enhance neuroplasticity during post-stroke rehabilitation $^{27}$. Several studies showed that an increase of the excitability in the stroke-affected ipsilesional M1 by electrical devices resulted in improved motor outcome ${ }^{28-30}$. The mechanisms of action of these techniques are under investigation but might involve changes in synaptic activity, gene expression and increases in neurotransmitter, receptor and neurotrophin levels ${ }^{27}$ or even enhanced fiber sprouting $^{31}$. A study of patients with stroke who had reached a plateau in motor recovery found that the volume of primary sensorimotor cortex activation in the ipsilesional hemisphere during affected hand movement was related to the level of behavioral recovery ${ }^{32}$.

\section{Three Major Phases of Stroke Reaction and Repair ${ }^{33}$}

Neuronal reorganization and plasticity that follow stroke begin in the very early stages, continue for several weeks, and involve brain regions distant to the affected site. Imaging studies (PET, EEG and fMRI) have revealed widespread changes in patterns of brain activation during simple movements of the affected hand after stroke; these alterations evolve over a timescale that is consistent with a gradual reorganization of the sensorimotor system.

1. The acute reaction to the injury and takes place in the initial hours when modifications become apparent in blood flow, edema, metabolism and inflammation.

2. A repair related epoch starts in the first days post 


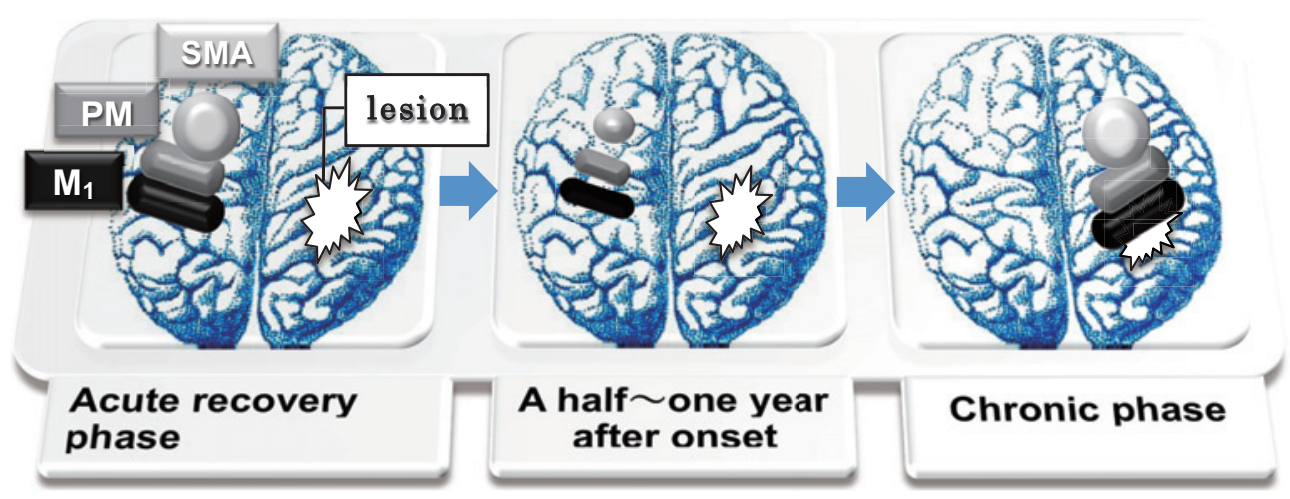

Fig. 1 Physiological recovery mechanism of hemiparesis.

Non-affected side motor related area facilitation induce the hemiparesis improvement at acute recovery phase ${ }^{21}$. Non-affected side motor related area cortex activity decreased at half and one year after stroke onset ${ }^{39}$. Finally affected side motor related area cortex activity increase is important for recovery. (SMA; supplementary motor area, $\mathrm{PM}$; premotor area, $\mathrm{M}_{1}$; primary motor area)

stroke and is on-going for several weeks. During this epoch spontaneous recovery is seen and endogenous repair related events reach their peak levels.

3. The third epoch begins weeks to months after stroke when spontaneous recovery has reached a plateau and represents a stable but still modifiable chronic phase.

Forms of Reaction to Stroke in Areas Which before

\section{Stroke Formed a Distributed Network}

For remodeling and recruitment of areas three main forms of reorganization have been described: (1) increased cortical excitability in cortical regions distant from, but connected to the stroke core; (2) reduced lateralized activation; and (3) somatotopic modifications within intact cortical regions ${ }^{25}$.

1. Increased functional activity occurs in several cortical areas which include motor, language, attention and visual functions ${ }^{23,34,35}$. Widespread areas of cortical hyperactivity appear days after stroke and diminish within months post incident ${ }^{36}$.

2. Reduced lateralized activation reflects the increased activity in the contralesional hemisphere, which reduces the extent of inter hemispheric balance as demonstrated in many stroke studies ${ }^{37,38}$. A subtype of the described increased activity as described in the first form or a passive event reflecting a reduced interhemispheric inhibition resulting from the stroke. Both phenomena, increased cortical excitability and reduced laterality, are related to spontaneous functional recovery ${ }^{35}$. Both are time dependent, increasing in the early weeks after stroke and decreasing over months thereafter. This decrease is greater among stroke patients with stronger functional recovery while the persistent increased activity over both hemispheres is greatest in those patients with the poorest outcome $e^{33,39}$.
3. Somatotopic reorganization which implies that intact cortical regions-in particular within the perinfarct area-reassign their functions which they subserved before stroke and take over function, which have been affected or lost by the ischemic event. Some studies suggest that the largest degree of somatotopic reorganization is associated with very large stroke injuries ${ }^{33}$. Such map shifts occur in primary and secondary cortical areas ${ }^{40}$.

The schema of physiological recovery mechanism of hemiparesis in strokes is showed in Figure $\mathbf{1 .}$

\section{NIBS in Neuro-rehabilitation}

Cortical brain stimulation is a novel technology that may show promise for improving motor recovery in the affected upper limb of stroke survivors most probably when it is combined with intense motor relearning. The idea of using magnetic or electrical stimulation to facilitate neural reorganization may be new, but the concepts have their origins in the very basic science techniques that have been used to understand neuroplasticity.

\section{TMS and Connectivity}

Facilitatory stimulation of ipsilesional M1 increases M1-supplementary motor area functional connectivity while inhibitory stimulation of contralesional M1 decreases contralesional but strengthens ipsilesional connectivity - a pattern that is associated with improved motor performance $^{39,41}$. Additionally, stimulation of regions other than M1 also induces substantial connectivity changes in inter-connected brain regions.

Transcranial direct current stimulation (tDCS) applied over a specific region induces distant effects on network connectivity, which may conceivably impact behavior. Modulation of distant neural regions via location-specific 

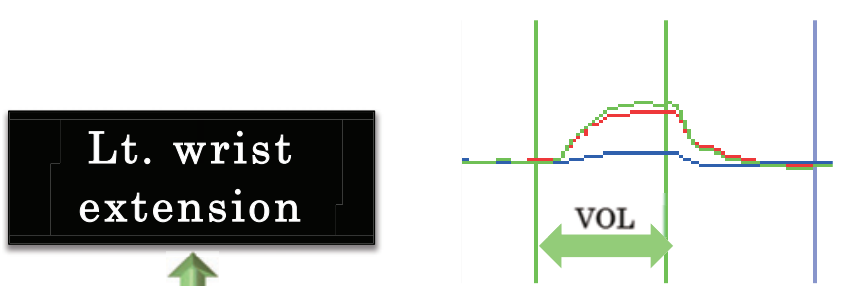

\section{Lt. ECRL ES}

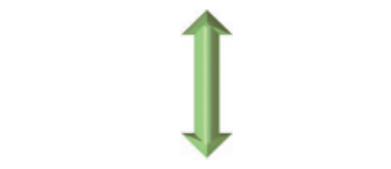

Lt. ECRL EMG-FES

Fig. 2 NIRS wave-form at rt. SMC area (cerebral infarction 57 years old female with lt. hemiparesis) ${ }^{65}$

Increased cerebral blood flow in the sensory-motor cortex area on the injured side during EMG-FES session compared to simple active movement or simple electrical stimulation in a NIRS study to noninvasively and dynamically measure hemoglobin levels in the brain during functional activity. (ECRL; extensor carpi radialis longs, VOL; voluntary movement, ES; electrical stimulation, FES; EMG-controlled FES)

stimulation holds intriguing possibilities. However, caution is urged when interpreting these preliminary results, since within this handful of studies, there is great variability in the experimental designs used (e.g., in the stimulation montage, period of stimulation, recording method, time of recording, type of analysis performed). In addition, there is significant inter-individual variability in results depending on the state of the subject's or network's activity (state-dependency), and the task performed.

Given these neuroimaging patterns after stroke, it has been proposed that upregulation of activity in the ipsilesional M1 or downregulation in the contralesional M1 might contribute to improved motor control ${ }^{42}$. Numerous proof of principle studies have now been done with some reporting that increasing excitability in ipsilesional M1 through high frequency repetitive transcranial magnetic stimulation (rTMS) or anodal tDCS may yield improvements in motor performance or motor learning in healthy subjects ${ }^{43,44}$ and small clinical studies have demonstrated modest, yet variable, improvements in individuals with stroke ${ }^{45-49}$. Importantly for rehabilitation, it has been proposed that some of these changes outlast the period of stimulation ${ }^{50,51}$. Similarly, downregulating excitability in the contralesional motor cortex in chronic stroke patients was also associated with improvements in motor function, along with increased cortical motor excitability in the ipsilesional M1 and decreased cortical excitability in the contralesional $\mathrm{M}^{52-54}$. Consistently, low frequency rTMS or cathodal tDCS applied to downregulate excitability in the contralesional hemisphere resulted in motor gains. When applied for this purpose, single sessions of 10-25 min of rTMS over the contralesional M1 were reported to induce improvements in movement kinematics $^{52,55-59}$.

\section{Role of Functional Electrical Stimulation (FES) in Brain Plasticity ${ }^{60}$}

FES is able to change axonal conduction velocities, axonal growth, and the myelination of peripheral nerves ${ }^{61}$. But the role of FES in the plasticity of the central nervous system (CNS) remains unclear. FES did not lead to acute neuroprotective events, highlighting the importance of cortical plasticity in the adjacent preserved regions of the ipsilateral cortex and its influence on behavioral motor recovery in rats ${ }^{62}$. Previous animal and clinical studies 


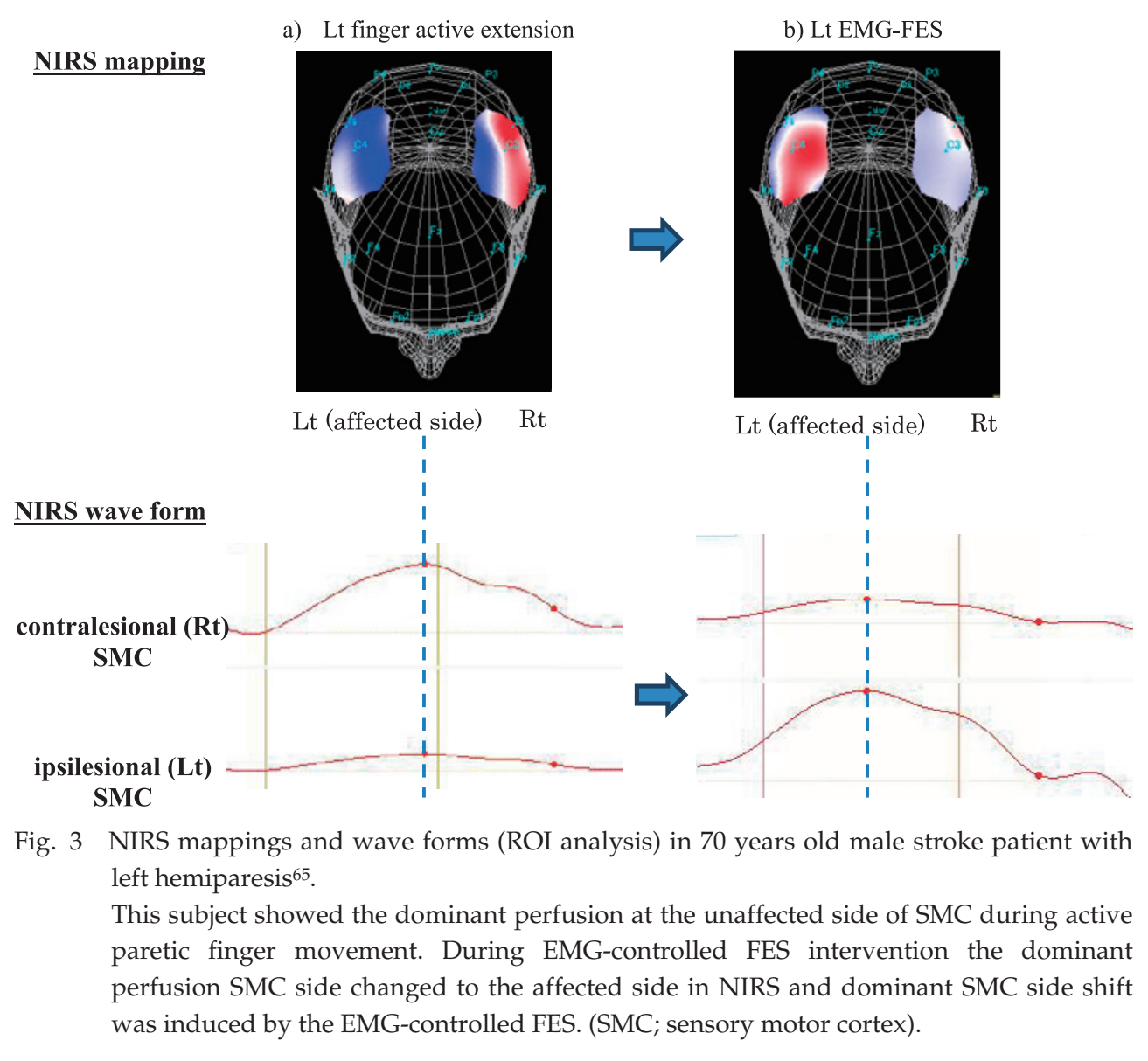

support the hypothesis that therapies that temporally link motor output and sensory feedback from the affected limb may facilitate neuroplastic changes leading to motor recovery. The Hebbian mechanisms believed to underlie activity dependent cortical reorganization suggest that rehabilitation therapies that repeatedly generate synchronous presynaptic and postsynaptic neural activity along motor and sensory pathways might facilitate synaptic remodeling, leading to neural reorganization and motor recovery ${ }^{62}$. FES maximizes the motor output (peripheral efferent activation) and corresponding muscle and joint proprioceptive feedback (afferent activation) that are tightly coupled and coordinated with movement by $\mathrm{FES}^{63}$.

There has been no study of brain activity during a therapeutic FES intervention because electrical stimulation obstructs assessment of brain activity via fMRI, single-photon emission computed tomography (SPECT), and PET and a usual FES intervention is difficult under those functional neuroimaging condition. However, NIRS is a recently developed neuroimaging methodology that is not influenced by electrical stimulation, and thus can be used to assess brain activity during FES in the rehabilitation room. The advantages of NIRS include its non- invasiveness, portability, the natural setting of the examination, low running cost and high sensitivity. NIRS measures changes in oxygenated and deoxygenated hemoglobin concentration that have been shown to correspond to regional cerebral blood flow $(\mathrm{CBF})^{64}$ and are thus interpreted as reflecting cortical activation. Hara $Y$ et al. $^{65}$ examined the effects of electromyography (EMG)controlled FES (EMG-FES) which induces greater muscle contraction by electrical stimulation in proportion to the integrated picked up EMG signal on cortical perfusion. There was an increase in the spatial extent of activation in the sensory motor cortex (SMC) in stroke patients during and after the EMG-FES therapy. Figure 2 shows a typical NIRS wave-form in the ipsilesional SMC from a 57 years old female stroke patient. There was more Oxy$\mathrm{Hb}$ concentration in the ipsilesional SMC during EMGFES than during voluntary movement only. $\mathrm{Oxy}-\mathrm{Hb}$ concentration levels in the SMC area were very low during simple electrical stimulation without voluntary movement. Prior to EMG-FES treatment, most subjects showed dominant perfusion in the contralesional SMC (Fig. 3a). After EMG-FES treatment, the SMC with dominant perfusion tended to change to the ipsilesional side (Fig. 3b); however, this was variable across subjects. In a few sub- 


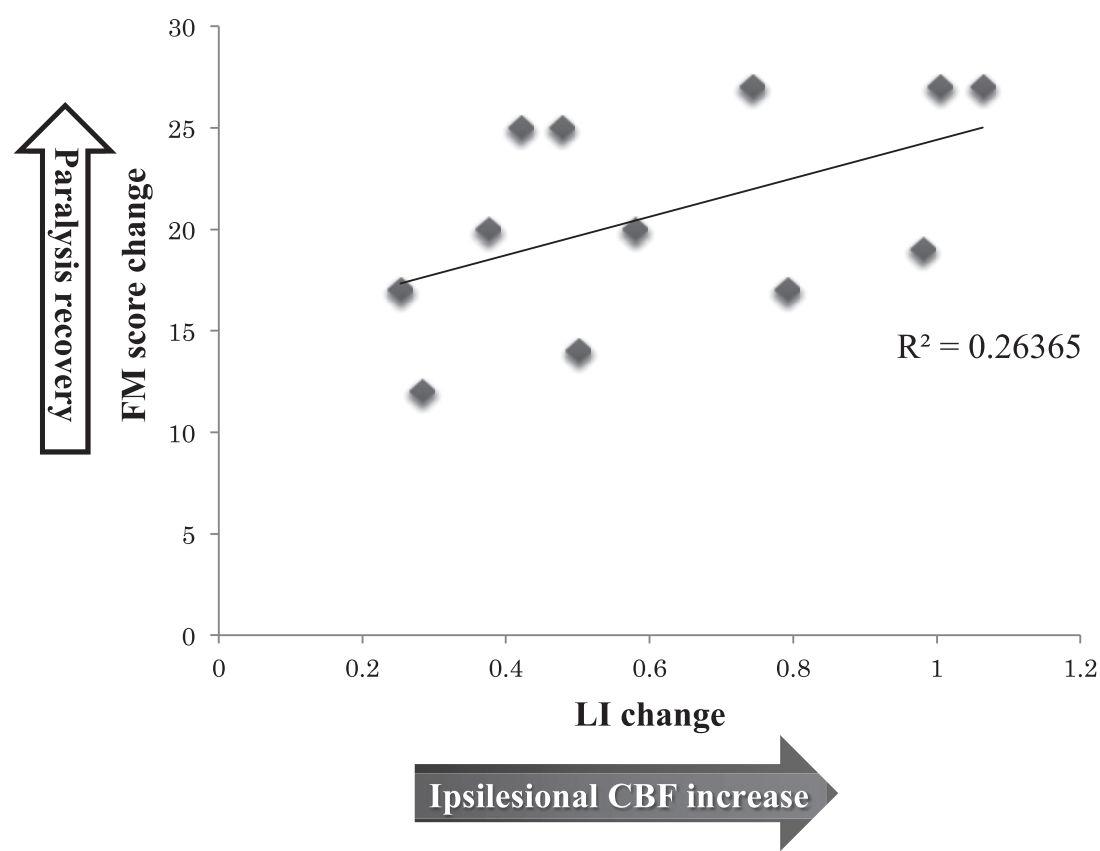

Fig. 4 Correlation between the FM score increase and laterality index change (active motion before and after EMG-FES rehabilitation) in SMC. $(n=12)^{65}$ LI change (VOL LI before and after EMG-FES) for SMC showed mildly strong positive correlation with FM score change (FM score before and after 5 months EMG-FES therapy). Trend line includes the Pearson product moment correlation coefficient and $\mathrm{P}$ value. 12 subjects showed the dominant perfusion at the unaffected side of SMC during active paretic finger movement. Their bilateral cortical activity was strongly lateralized towards the ipsilesional hemisphere during EMG-FES. A greater improvement in FM score was associated with a larger increase in affected side SMC. Only patients with a large increase in SMC activation showed a significant improvement in the FM score, whereas those with less SMC activation increase had less functional benefit. (FM score; Fugle Myer score, LI; Laterality Index, VOL; Voluntary movement)

jects, the ipsilesional SMC already exhibited an increase in perfusion prior to EMG-FES treatment. The increase in ipsilesional SMC perfusion and the functional improvement of upper extremity hemiparesis after EMG-FES was less in these subjects. It is hypothesized that these subjects had already achieved brain reorganization in the ipsilesional SMC prior to EMG-FES treatment with usual brain functional recovery or/and usual rehabilitation. After EMG-FES treatment, ipsilesional SMC perfusion and the functional improvement of upper extremity hemiparesis occurred only in patients who had sufficient potential for brain reorganization in the ipsilesional SMC. The relation between increases in cortical perfusion and functional upper extremity recovery in chronic stroke patients with moderate to severe hemiparesis was examined. This study demonstrated that 5 months of EMGFES treatment induced functional recovery in the hemiparetic hand of chronic stroke patients that was associ- ated with increases in cortical perfusion patterns (Fig. 4). The results indicate that the sensory motor integration due to EMG-FES may facilitate the perfusion of the ipsilesional SMC and result in functional improvement of hemiparetic upper extremity.

Early in the recovery of finger and hand movements after stroke with mild to moderate hemiparesis there is a bilateral cortical activation pattern, which becomes progressively lateralized to the affected cerebral hemisphere as hand function normalizes ${ }^{66}$. However, the physiologic explanation for the transient lack of movement-related activity in the affected hemisphere in the early chronic stage of recovery in this study is not clear. We observed a transient lack of activation in the affected hemisphere despite clinical improvement of hand function. The movement-related increase in $\mathrm{Oxy}-\mathrm{Hb}$ activity reappeared after further improvement in the chronic stage ${ }^{67}$. The patterns of cortical activity in our patients support 
the view that recovery from hemiparetic stroke depends on the recruitment of alternative systems even in the affected hemisphere ${ }^{68,69}$. However, as observed previously in individual patients ${ }^{70}$, there were large inter-individual differences in cortical activity related to movement of the affected hand. These differences were probably due to differences in lesion location and extent, differences in performance, and possibly differences in functional ability $^{67}$. Patients with excellent recovery of motor function after stroke activate the primary motor and premotor cortices bilaterally when executing a task with the affected hand ${ }^{22}$. Lewinski et al. ${ }^{71}$ reported that clinical improvement was accompanied by an increase in ipsilesional SMC activation, as revealed by fMRI, and enhanced intracortical facilitation, as revealed by paired transcranial magnetic stimulation. Our study ${ }^{65}$ was therefore in accordance with previous reports ${ }^{72}$, and support the notion that EMG-FES is more effective for increasing activation of the ipsilesional SMC in patients with severe to moderate motor impairment than active voluntary motion and simple electrical stimulation. The fact that the degree of clinical improvement after treatment with EMG-FES was paralleled by changes in cortical activation supports the assumption that treatment-driven clinical improvements in chronic stroke patients are directly related to a reorganization at the cortical level, as previously reported ${ }^{73-76}$.

Stinear et al. $^{77}$ reported that meaningful functional improvements can occur after 1 month of rehabilitation performed within the first 3 years after stroke, a considerably longer interval than commonly accepted. Our study showed that the functional improvement in chronic stroke patients is possible after 5 months of EMG-FES rehabilitation. Bhatt et al. $^{78}$ reported that finger tracking combined with electrical stimulation may have more influence on brain reorganization than either treatment alone. As EMG-FES is a similar functional intervention to finger tracking combined with electrical stimulation, this novel FES intervention may have a more facilitative effect on brain activation than voluntary motion and simple electrical stimulation. The motor output and corresponding muscle and joint proprioceptive feedback may be tightly coupled and coordinated with movement by EMG-FES.

\section{Future Directions or Designing Optimal \\ Neuro-rehabilitation}

The presence of critical time windows for the application of growth and plasticity promoting agents and of training-dependent plasticity suggests that careful consid- eration of rehabilitation onset times, tailored training to the type and extent of stroke and the patient's history are required. Selection and stabilization of newly formed functional connections by rehabilitative training. An important point is that compensation also occurs during the post stroke sensitive period and is also mediated by plastic changes in peri-infarct cortex and other cortical area ${ }^{1,79}$. There is a unique milieu of enhanced plasticity for 1-3 months after ischemic stroke, and that within this time window both spontaneous and intervention-mediated recovery from impairment is maximal ${ }^{80}$.

In the last step of recovery that is based mainly on rehabilitative training the spared and the new circuitry of the CNS is shaped by selection and stabilization of functional connections and pruning of the non-functional ones. Hebbian learning rules might play a crucial role in this step in the sense that Hebbian plasticity mechanisms redistribute synaptic strength to favor the wiring of pathways that are coincidently active ${ }^{6}$.

Conflict of Interest: There is no conflict of interest to disclose.

\section{References}

1. Nudo RJ: Mechanisms for recovery of motor function following cortical damage. Curr Opin Neurobiol 2006; 16: 638-644.

2. Kolb B: Overview of cortical plasticity and recovery from brain injury. Phys Med Rehabil Clin North Am 2003; 14 (suppl): S7-25.

3. Harvey RL: Motor recovery after stroke: new directions in scientific inquiry. Top Stroke Rehabil 2003; 14 (1S): S1-S5.

4. Nudo RJ, Wise BM, SiFuentes F, Milliken GW: Neural substrates for the effects of rehabilitative training on motor recovery after ischemic infarct. Science 1996; 272: 1791-1794.

5. Nudo RJ, Milliken GW, Jenkins GW, Merzenich MM: Usedependent alterations of movement representations in primary motor cortex of adult squirrel monkeys. J Neurosci 1996; 16: 785-807.

6. Murphy $\mathrm{TH}$, Corbett D: Plasticity during stroke recovery: from synapse to behavior. Nat Rev Neurosci 2009; 10: 861-872.

7. Takatsuru Y, Fukumoto D, Yoshitomo M, Nemoto T, Tsukada $\mathrm{H}$, Nabekura J: Neuronal circuit remodeling in the contralateral cortical hemisphere during functional recovery from cerebral infarction. J Neurosci 2009; 29: 1008110086.

8. Dijkhuizen R, Ren J, Mandeville J, et al.: Functional magnetic resonance imaging of reorganization in rat brain after stroke. Proc Natl Acad Sci USA 2001; 98: 12766-12771.

9. Dijkhuizen R, Singhal A, Mandeville J, et al.: Correlation between brain reorganization, ischemic damage, and neurologic status after transient focal cerebral ischemia in rats: a functional magnetic resonance imaging study. J Neurosci 2003; 23: 510-517.

10. Hsu J, Jones T: Contralesional neural plasticity and functional changes in the less-affected forelimb after large and small cortical infarcts in rats. Exp Neurol 2006; 201: 479- 
494.

11. Van Meer MP, Otte WM, van der Marel K, et al.: Extent of bilateral neuronal network reorganization and functional recovery in relation to stroke severity. J Neurosci 2012; 32: 4495-4507.

12. Starkey ML, Bleul C, Zorner B, et al.: Back seat driving: hindlimb corticospinal neurons assume forelimb control following ischaemic stroke. Brain 2012; 135 (Pt. 11): 32653281.

13. Biernaskie J, Chernenko G, Corbett D: Efficacy of rehabilitative experience declines with time after focal ischemic brain injury. J Neurosci 2004; 24: 1245-1254.

14. Wahl AS, Omlor W, Rubio JC, et al.: A synchronous therapy restores motor control by rewiring of the rat corticospinal tract after stroke. Science 2014; 344: 1250-1255.

15. Calautti C, Leroy F, Guincestre JY, Baron JC: Dynamics of motor network overactivation after striatocapsular stroke: a longitudinal PET study using a fixed-performance paradigm. Stroke 2001; 32: 2534-2542.

16. Nhan $\mathrm{H}$, Barquist $\mathrm{K}$, Bell $\mathrm{K}$, et al.: Brain function early after stroke in relation to subsequent recovery. J Cereb Blood Flow Metab 2004; 24: 756-763.

17. Binkofski F, Seitz R, Arnold S, et al.: Thalamic metabolism and corticospinal tract integrity determine motor recovery in stroke. Ann Neurol 1996; 39: 460-470.

18. Carmichael ST, Tatsukawa K, Katsman D, et al.: Evolution of diaschisis in a focal stroke model. Stroke 2004; 35: 758763.

19. Seitz R, Azari N, Knorr U, et al.: The role of diaschisis in stroke recovery. Stroke 1999; 30: 1844-1850.

20. Nishibe M, Urban ET 3rd, Barbay S, Nudo RJ: Rehabilitative Training Promotes Rapid Motor Recovery but Delayed Motor Map Reorganization in a Rat Cortical Ischemic Infarct Model. Neurorehabil Neural Repair 2014 Jul 22. pii: 1545968314543499. [Epub ahead of print].

21. Calautti C, Baron JC: Functional neuroimaging studies of motor recovery after stroke in adults: a review. Stroke 2003; 34: 1553-1566.

22. Bütefisch $\mathrm{CM}$, Kleiser $\mathrm{R}$, Körber $\mathrm{B}$, et al.: Recruitment of contralesional motor cortex in stroke patients with recovery of hand function. Neurol 2005; 64: 1067-1069.

23. Chollet F, DiPiero V, Wise RJ, Brooks D, Dolan RJ, Frackowiak RS: The functional anatomy of motor recovery after stroke in humans: a study with positron emission tomography. Ann Neurol 1991; 29: 63-71.

24. European Stroke Organisation (ESO) Executive Committee; ESO Writing Committee: Guidelines for management of ischaemic stroke and transient ischaemic attack 2008. Cerebrovasc Dis 2008; 25: 457-507.

25. Wahl AS, Schwab ME: Finding an optimal rehabilitation paradigm after stroke: enhancing fiber growth and training of the brain at the right moment. Front Hum Neurosci 2014; 8: 1-13.

26. Krakauer JW, Carmichael ST, Corbett D, Wittenberg GF: Getting neurorehabilitation right: what can be learned from animal models? Neurorehabil Neural Repair 2012; 26: 923-931.

27. Dimyan MA, Cohen LG: Neuroplasticity in the context of motor rehabilitation after stroke. Nat Rev Neurol 2011; 7: 76-85.

28. Malcolm MP, Triggs WJ, Light KE, et al.: Repetitive transcranial magnetic stimulation as an adjunct to constraintinduced therapy: an exploratory randomized controlled trial. Am J Phys Med Rehabil 2007; 86: 707-715.

29. Ameli M, Grefkes C, Kemper F, et al.: Differential effects of high-frequency repetitive transcranial magnetic stimu- lation over ipsilesional primary motor cortex in cortical and subcortical middle cerebral artery stroke. Ann Neurol 2009; 66: 298-309.

30. Koganemaru S, Mima T, Thabit MN, et al.: Recovery of upper-limb function due to enhanced use-dependent plasticity in chronic stroke patients. Brain 2010; 133: 33733384.

31. Martin JH: Systems neurobiology of restorative neurology and future directions for repair of the damaged motor systems. Clin Neurol Neurosurg 2012; 114: 515-523.

32. Zemke A, Heagerty P, Lee C, Cramer S: Motor cortex organization after stroke is related to side of stroke and level of recovery. Stroke 2003; 34: E23-E28.

33. Cramer SC, Crafton KR: Somatotopy and movement representation sites following cortical stroke. Exp Brain Res 2006; 168: 25-32.

34. Brion JP, Demeurisse G, Capon A: Evidence of cortical reorganization in hemiparetic patients. Stroke 1989; 20: 1079-1084.

35. Cramer SC: Repairing the human brain after stroke: I. Mechanisms of spontaneous recovery. Ann Neurol 2008; 63: 272-287.

36. Ward NS: Functional reorganization of the cerebral motor system after stroke. Curr Opin Neurol 2004; 17: 725-730.

37. Weiller C, Ramsay SC, Wise RJ, Friston KJ, Frackowiak RS: Individual patterns of functional reorganization in the human cerebral cortex after capsular infarction. Ann Neurol 1993; 33: 181-189.

38. Seitz RJ, Höflich P, Binkofski F, Tellmann L, Herzog H, Freund HJ: Role of the premotor cortex in recovery from middle cerebral artery infarction. Arch Neurol 1998; 55: 1081-1088.

39. Ward NS, Brown MM, Thompson AJ, Frackowiak RS: Neural correlates of motor recovery after stroke: a longitudinal fMRI study. Brain 2003; 126 (Pt. 11): 2476-2496.

40. Byrnes ML, Thickbroom GW, Phillips BA, Mastaglia FL: Long-term changes in motor cortical organization after recovery from subcortical stroke. Brain Res 2001; 889: 278287.

41. Rehme AK, Eickhoff SB, Wang LE, Fink GR, Grefkes C: Dynamic causal modeling of cortical activity from the acute to the chronic stage after stroke. Neuroimage 2011; 55: 1147-1158.

42. Ward NS, Cohen LG: Mechanisms underlying recovery of motor function after stroke. [Review]. Arch Neurol 2004; 61: 1844-1848.

43. Nitsche MA, Nitsche MS, Klein CC, Tergau F, Rothwell JC, Paulus W: Level of action of cathodal DC polarization induced inhibition of the human motor cortex. Clin Neurophysiol 2003; 114: 600-604.

44. Reis J, Schambra HM, Cohen LG, et al.: Noninvasive cortical stimulation enhances motor skill acquisition over multiple days through an effect on consolidation. Proc Natl Acad Sci USA 2009; 106: 1590-1595.

45. Hummel F, Cohen LG: Improvement of motor function with noninvasive cortical stimulation in a patient with chronic stroke. Neurorehabil Neural Repair 2005; 19: 1419.

46. Hummel FC, Cohen LG: Non-invasive brain stimulation: a new strategy to improve neurorehabilitation after stroke? Lancet Neurol 2006; 5: 708-712.

47. Khedr EM, Ahmed MA, Fathy N, Rothwell JC: Therapeutic trial of repetitive transcranial magnetic stimulation after acute ischemic stroke. Neurology 2005; 65: 466-468.

48. Sandrini M, Cohen LG: Noninvasive brain stimulation in neurorehabilitation. Handb Clin Neurol 2013; 116C: 499- 
524.

49. Pomeroy VM, Cloud G, Tallis RC, Donaldson C, Nayak V, Miller S: Transcranial magnetic stimulation and muscle contraction to enhance stroke recovery: a randomized proof-of-principle and feasibility investigation. Neurorehabil Neural Repair 2007; 21: 509-517.

50. Khedr EM, Etraby AE, Hemeda M, Nasef AM, Razek AA: Long-term effect of repetitive transcranial magnetic stimulation on motor function recovery after acute ischemic stroke. Acta Neurol Scand 2010; 121: 30-37.

51. Krawczyk DC: The cognition and neuroscience of relational reasoning. Brain Res 2012; 1428: 13-23.

52. Takeuchi N, Chuma T, Matsuo Y, Watanabe I, Ikoma K: Repetitive transcranial magnetic stimulation of contralesional primary motor cortex improves hand function after stroke. Stroke 2005; 36: 2681-2686.

53. Takeuchi N, Tada T, Toshima M, Chuma T, Matsuo Y, Ikoma K: Inhibition of the unaffected motor cortex by 1 $\mathrm{Hz}$ repetitive transcranical magnetic stimulation enhances motor performance and training effect of the paretic hand inpatients with chronic stroke. J Rehabil Med 2008; 40: 298-303.

54. Fregni F, Pascual-Leone A: Hand motor recovery after stroke: tuning the orchestra to improve hand motor function. Cogn Behav Neurol 2006; 19: 21-33.

55. Mansur CG, Fregni F, Boggio PS, et al.: A sham stimulation-controlled trial of rTMS of the unaffected hemisphere in stroke patients. Neurology 2005; 64: 18021804.

56. Boggio PS, Castro LO, Savagim EA, et al.: Enhancement of non-dominant hand motor function by anodal transcranial direct current stimulation. Neurosci Lett 2006; 404: 232-236.

57. Dafotakis M, Grefkes C, Eickhoff SB, Karbe H, Fink GR, Nowak DA: Effects of rTMS on grip force control following subcortical stroke. Exp Neurol 2008; 211: 407-412.

58. Nowak DA, Grefkes C, Dafotakis M, et al.: Effects of lowfrequency repetitive transcranial magnetic stimulation of the contralesional primary motor cortex on movement kinematics and neural activity in subcortical stroke. Arch Neurol 2008; 65: 741-747.

59. Liepert J, Zittel S, Weiller C: Improvement of dexterity by single session low-frequency repetitive transcranial magnetic stimulation over the contralesional motor cortex in acute stroke: a double-blind placebo-controlled cross over trial. Restor Neurol Neurosci 2007; 25: 461-465.

60. Liew S, Garrison K, Werner J, Aziz-Zadeh L: The mirror neuron system: innovations and implications for occupational therapy. OTJR: Occupation, participation and health 2012; 32: 79-86.

61. Al-Majed A, Neumann C, Brushart T, et al.: Brief electrical stimulation promotes the speed and accuracy of motor axonal regeneration. J Neurosci 2000; 20: 2602-2608.

62. Cecatto RB, Maximino JR, Chadi G: Motor recovery and cortical plasticity after functional electrical stimulation in a rat model of focal stroke. Am J Phys Med Rehabil 2014; 93: 791-800.

63. Rushton DN: Functional electrical stimulation and rehabilitation. -A hypothesis. Med Eng Phys 2003; 25: 75-78.

64. Toronov V, Webb A, Choi JH, Wolf M, Michalos A, Gratton E: Investigation of human brain hemodynamics by simultaneous near-infrared spectroscopy and functional magnetic resonance imaging. Med Phys 2001; 28: 521-527.

65. Hara Y, Obayashi S, Tsujiuchi K, Muraoka Y: The effects of electromyography controlled functional electrical stimulation on upper extremity function and cortical perfusion in stroke patients. Clin Neurophysiol 2013; 124: 2008-2015.

66. Takeda K, Gomi Y, Imai I, Shimoda N, Hiwatari M, Kato $\mathrm{H}$ : Shift of motor activation areas during recovery from hemiparesis after cerebral infarction: A longitudinal study with near-infrared spectroscopy. Neurosci Res 2007; 59: 136-144.

67. Binkofski F, Seitz RJ: Modulation of the BOLD-response in early recovery from sensorimotor stroke. Neurology 2004; 63: 1223-1229.

68. Calautti C, Leroy F, Guincestre JY, Baron JC: Dynamics of motor network overactivation after striatocapsular stroke: a longitudinal PET study using a fixed-performance paradigm. Stroke 2001; 32: 2534-2542.

69. Johansen-Berg H, Rushworth MF, Bogdanovic MD, Kischka U, Wimalaratna S, Matthews PM: The role of ipsilateral premotor cortex in hand movement after stroke. Proc Natl Acad Sci USA 2002; 99: 14518-14523.

70. Weiller C, Ramsay SC, Wise RJ, Friston KJ, Frackowiak RS: Individual patterns of functional reorganization in the human cerebral cortex after capsular infarction. Ann Neurol 1993; 33: 181-189.

71. Lewinski FV, Hofer S, Kaus J, et al.: Efficacy of EMGtriggered electrical arm stimulation in chronic hemiparetic stroke patients. Restor Neurol Neurosci 2009; 27: 189-197.

72. Chae J, Yu D: A critical review of neuromuscular electrical stimulation for treatment of motor dysfunction in hemiplegia. Assist Technol 2000; 12: 33-49.

73. Carey JR, Kimberley TJ, Lewis SM, et al.: Analysis of fMRI and finger tracking training in subjects with chronic stroke. Brain 2002; 125: 773-788.

74. Liepert J, Bauder H, Wolfgang HR, Miltner WH, Taub E, Weiller C: Treatment-induced cortical reorganization after stroke in humans. Stroke 2000; 31: 1210-1216.

75. Koski L, Mernar TJ, Dobkin BH: Immediate and longterm changes in corticomotor output in response to rehabilitation: correlation with functional improvements in chronic stroke. Neurorehabil Neural Repair 2004; 18: 230249.

76. Platz T, van Kaick S, Moller L, Freund S, Winter T, Kim $\mathrm{IH}$ : Impairment-oriented training and adaptive motor cortex reorganisation after stroke: a fTMS study. J Neurol 2005; 252: 1363-1371.

77. Stinear CM, Barber PA, Smale PR, Coxon JP, Fleming MK, Byblow WD: Functional potential in chronic stroke patients depends on corticospinal tract integrity. Brain 2007; 130: 170-180.

78. Bhatt E, Nagpal A, Greer KH, et al.: Effect of finger tracking combined with electrical stimulation on brain reorganization and hand function in subjects with stroke. Exp Brain Res 2007; 182: 435-447.

79. Gharbawie OA, Karl JM, Whishaw IO: Recovery of skilled reaching following motor cortex stroke: do residual corticofugal fibers mediate compensatory recovery? Eur J Neurosci 2007; 26: 3309-3327.

80. Zeiler SR, Krakauer JW: The interaction between training and plasticity in the post stroke brain. Curr Opin Neurol 2013; 26: 609-616.

(Received, November 11, 2014)

(Accepted, December 22, 2014) 